\title{
MAGNETO-ELASTIC COMBINED MULTIDIMENSIONAL RESONANCE OF A ROTATING CIRCULAR PLATE IN A DOUBLE-DIRECTION MAGNETIC FIELD
}

\author{
Zhe Li, Yu Da Hu, Wen Qiang Li \\ School of Civil Engineering and Mechanics, Yanshan University, Qinhuangdao, China, and \\ Hebei Key Laboratory of Mechanical Reliability for Heavy Equipments and Large Structures, Yanshan University, \\ Qinhuangdao,China; e-mail: huyuda03@163.com
}

\begin{abstract}
Magneto-elastic nonlinear non-axisymmetric resonance is investigated for a rotating annular plate in a double-direction magnetic field. According to transverse and longitudinal magneto-elastic non-axisymmetric vibration equations of the thin annular plate, and considering the influence of the static load term, non-axisymmetric vibration differential equations by combined parametric and forced excitations are obtained through application of the Galerkin method. Then, the method of multiple scales is applied to solve differential vibration equations. By numerical computations, the influence of magnetic induction intensity, inner and outer diameters, excitation and radial forces on transverse and longitudinal resonance characteristics are analyzed.
\end{abstract}

Keywords: magneto-elastic, annular plate, multi-dimensional vibration, combination resonance, the method of multiple scales

\section{Introduction}

Rotating annular components are widely used in aerospace, large generator sets, mechanical devices accompanied by impacts of complex environment. A change of system initial conditions and physical parameters of an annular plate in complex environment such as mechanical fields may lead to large vibration and instability of the annular plate and damage the system. With the development of modern industry, in order to make hard disks have a faster reading speed, the cutting speed of the machine blade should be increased and precision of the finished product improved. The security and stability requirements also increase. When a rotating structure is affected by external forces, it will produce corresponding vibration modes and noise pollution, accelerate wear of machine components, waste energy, and reduce the working life and efficiency of the machine, which is very adverse to the operation of the machine. Mechanical components will be damaged, and even endanger personal safety. Therefore, it is valuable and meaningful to study the vibration of rotating annular plates.

Many researchers have done a lot of theoretical research on magneto-elastic vibration of structures. Narain and Srivastava (2004) studied magneto-elastic torsional vibration of a non-homogeneous aeolotropic cylindrical shell made of viscoelastic solids and derived the frequency equation of the shell. Hu and Wang (2016), Hu and Li (2017, 2018) and Hu et al. (2018a) studied vibration of a rotating circular plate in a magnetic field, established electromagnetic nonliner vibration equations and analyzed principal parametric and combined resonances. Meanwhile, bifurcation, chaos and free vibration under static loads were discussed, and the influence of a variable parameter on the resonance characteristics of the system was analyzed. Li et al. (2017) studied nonlinear principal parameter vibration and stability of a rotating circular plate 
with variable speed in a magnetic field and derived the magneto-elastic vibration equation of a rotating circular plate in a magnetic field. The resonance amplitude response equation of the nonlinear principal parameter was obtained by using the multi-scale method, and the effects of parameters on the system stability were discussed.

As the problem of rotating plates, the annular plate is also a subject studied by scholars. Chen and Chen (2007) analyzed non-axisymmetric vibration and stability of rotating sandwich annular plates by means of the finite element method, and the effects of stiffness and thickness on vibration of the system were discussed. Maretic et al. (2007) studied transverse vibration of an annular plate under different boundary conditions, analyzed the influence of different parameters and boundary conditions on the vibration frequency, and determined the instability critical value of angular velocity and moment of the annular plate through stability analysis. Yeh (2011) discussed free vibration of rotating annular plates with electrorheological damping. The natural frequencies and loss factors of the system were obtained by solving complex eigenvalues of the system. Younesian et al. (2015) studied vibration of a hollow annular plate under the action of a rotating force. The finite element model was established by using the Galerkin method, and the model was verified by computer simulation. Kang (2017) studied axisymmetric free vibration of rotating annular plates with variable thickness by using the Ritz method. The stress, strain and radial displacement of rotating annular plates were obtained by calculation. At the same time, the natural frequencies and modes of rotating nonuniform annular plates with different angular velocities and internal and external diameters were given.

Aiming at the parametric vibration and combined vibration of the system, Hu et al. (2018b) studied the resonance of the principal parameter of the axial moving beam under a static load and analyzed the influence of variation of the parameters on the resonance characteristics of the system. Wu et al. (2007) provided equations of motion of a cable and studied the effect of periodic excitation on nonlinear parametric vibration of the cable. Xia et al. (2011) used the finite element method to study nonlinear vibration of a cable-beam system under simple harmonic and random loads and compared it with the analytical solution and experimentally verified the correctness of the finite element method. Tylikowski and Frischmuth (2003) analyzed stability of a circular plate parameter vibration under the action of a plane force with the Lyapunov method and analyzed the stability problem of the circular plate. Awrejcewicz et al. (2004, 2006) studied parametric vibration of flexible plates and transformed the solution into partial differential equations to analyze the effect of parameter variation on vibration. Hu and Zhang (2013) analyzed the resonance problem of the main parameters of a rectangular plate and the influence of a periodic excitation force and axial velocity on stability of the system. Niu et al. (2012) studied dynamic characteristics of rectangular plates under an in-plane periodic loading. They established a partial differential equation based on a mechanical model, solved and analyzed the amplitude-frequency relation by using the harmonic balance method and studied stability of the solution as well as the stable region of the system. Ma et al. (2014) studied the principal parameter resonance of a plate under a moving boundary condition and discussed the amplitude-frequency and amplitude-excitation relation. Zhang and Huo (1991) studied the bifurcation problem of nonlinear systems under the combined action of parameter and forced excitation, and obtained many valuable conclusions.

On the basis of the magneto-elastic transverse and longitudinal vibration equations of rotating circular plates, the static load term is considered and differential equations of vibration by the combined parametric and forced excitations are derived. The resonance responses for the combination of the primary resonance-principal parameter resonance of different modes are obtained by means of the multi-scale method. By using a numerical example, the influence of different parameters on the resonance characteristics is analyzed. 


\section{Double-direction vibration equations of the rotating annular plate}

Consider a circular plate rotating in a double-direction magnetic field $B\left(B_{0 r}, 0, B_{0 z}\right)$ at a constant rotational angular speed $\Omega$. The plate is subjected to transverse load $P_{z}$ and radial force $F_{0 r}$, as shown in Fig. 1. We assume $F_{0 r}=F_{0}+F_{1} \cos \left(\omega_{1} t\right)$.

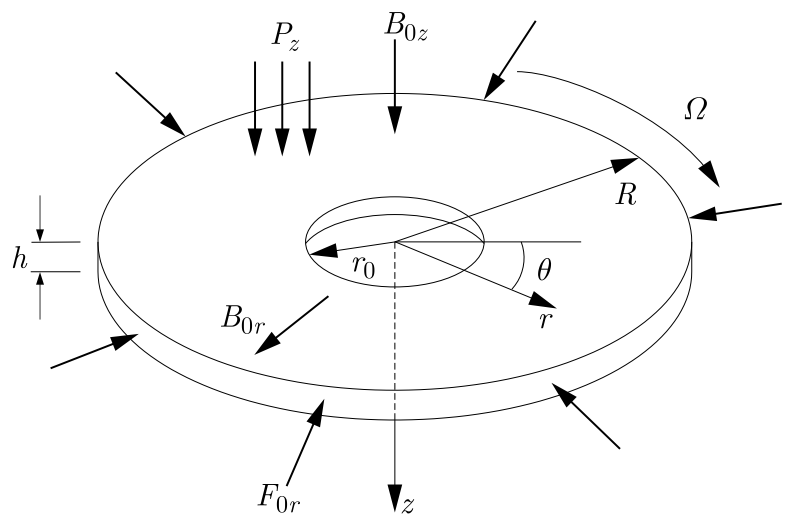

Fig. 1. The mechanical model of the rotating annular plate

Since the rotating annular plate studied in this paper is a symmetric structure and the boundary constraints and external loads are symmetric, the radial displacement $u_{r}$ is considered to be a function of $r$ while the toroidal displacement $u_{\theta}$ can be neglected. According to the expression of kinetic and strain energy, one can derive magneto-elastic nonlinear non-axisymmetric vibration equations of the spinning annular thin plate by using the Hamilton principle as follows (Hu and $\mathrm{Li}, 2017$ )

$$
\begin{aligned}
- & D_{M}\left(\frac{\partial^{4} w}{\partial r^{4}}+\frac{2}{r} \frac{\partial^{3} w}{\partial r^{3}}-\frac{1}{r^{2}} \frac{\partial^{2} w}{\partial r^{2}}+\frac{2}{r^{2}} \frac{\partial^{4} w}{\partial r^{2} \partial \theta^{2}}+\frac{1}{r^{3}} \frac{\partial w}{\partial r}-\frac{3}{r^{3}} \frac{\partial^{3} w}{\partial r \partial \theta^{2}}+\frac{4}{r^{4}} \frac{\partial^{2} w}{\partial \theta^{2}}+\frac{1}{r^{4}} \frac{\partial^{4} w}{\partial \theta^{4}}\right) \\
& +D_{N}\left[\frac{\mu+1}{r} \frac{\partial u_{r}}{\partial r} \frac{\partial w}{\partial r}+\frac{1}{2 r}\left(\frac{\partial w}{\partial r}\right)^{3}+\frac{2 \mu-1}{2 r^{3}} \frac{\partial w}{\partial r}\left(\frac{\partial w}{\partial \theta}\right)^{2}+\frac{\partial^{2} u_{r}}{\partial r^{2}} \frac{\partial w}{\partial r}+\frac{3}{2}\left(\frac{\partial w}{\partial r}\right)^{2} \frac{\partial^{2} w}{\partial r^{2}}\right. \\
& +\frac{2-\mu}{r^{2}} \frac{\partial w}{\partial r} \frac{\partial w}{\partial \theta} \frac{\partial^{2} w}{\partial r \partial \theta}+\frac{\partial u_{r}}{\partial r} \frac{\partial^{2} w}{\partial r^{2}}+\frac{\mu}{r} u_{r} \frac{\partial^{2} w}{\partial r^{2}}+\frac{1}{2 r^{2}}\left(\frac{\partial w}{\partial \theta}\right)^{2} \frac{\partial^{2} w}{\partial r^{2}}+\frac{1}{r^{3}} u_{r} \frac{\partial^{2} w}{\partial \theta^{2}} \\
& \left.+\frac{3}{2 r^{4}}\left(\frac{\partial w}{\partial \theta}\right)^{2} \frac{\partial^{2} w}{\partial \theta^{2}}+\frac{\mu}{r^{3}} \frac{\partial u_{r}}{\partial r} \frac{\partial^{2} w}{\partial \theta^{2}}+\frac{1}{2 r^{2}}\left(\frac{\partial w}{\partial r}\right)^{2} \frac{\partial^{2} w}{\partial \theta^{2}}+\frac{\mu}{r^{2}} \frac{\partial w}{\partial r} \frac{\partial^{2} w}{\partial r \partial \theta}\right] \\
& -\sigma_{0} h B_{0 r}\left[\left(\frac{\partial w}{\partial t}+\Omega \frac{\partial w}{\partial \theta}\right) B_{0 r}-\frac{d u_{r}}{d t} B_{0 z}\right]+\frac{\sigma_{0} h^{3}}{12} B_{0 z}^{2}\left(\frac{1}{r} \frac{\partial^{2} w}{\partial r \partial t}+\frac{\partial^{3} w}{\partial r^{2} \partial t}+\frac{1}{r^{2}} \frac{\partial^{3} w}{\partial r \partial \theta^{2}}\right) \\
& +\frac{\sigma_{0} h^{3}}{12} B_{0 r}^{2}\left(\frac{1}{r} \frac{\partial^{3} w}{\partial t \partial \theta^{2}}+\Omega \frac{\partial^{2} w}{\partial t \partial \theta}\right)+F_{0 r} \frac{\partial^{2} w}{\partial r^{2}}+P_{z}=\rho h\left(\frac{\partial^{2} w}{\partial t^{2}}+2 \Omega \frac{\partial^{2} w}{\partial t \partial \theta}+\Omega^{2} \frac{\partial^{2} w}{\partial \theta^{2}}\right) \\
& -\frac{\rho h^{3}}{12}\left[\left(\frac{1}{r} \frac{\partial^{3} w}{\partial r \partial t^{2}}+\frac{\partial^{4} w}{\partial r^{2} \partial t^{2}}+\frac{1}{r^{2}} \frac{\partial^{4} w}{\partial t^{2} \partial \theta^{2}}\right)+2 \Omega\left(\frac{1}{r} \frac{\partial^{3} w}{\partial t \partial \theta \partial r}+\frac{\partial^{4} w}{\partial t \partial \theta \partial r^{2}}+\frac{1}{r^{2}} \frac{\partial^{4} w}{\partial t \partial \theta^{3}}\right)\right. \\
& \left.+\Omega^{2}\left(\frac{1}{r} \frac{\partial^{3} w}{\partial r \partial \theta^{2}}+\frac{\partial^{4} w}{\partial r^{2} \partial \theta^{2}}+\frac{1}{r^{2}} \frac{\partial^{4} w}{\partial \theta^{4}}-\frac{1}{r} \frac{\partial w}{\partial r}-\frac{\partial^{2} w}{\partial r^{2}}-\frac{1}{r^{2}} \frac{\partial^{2} w}{\partial \theta^{2}}\right)\right] \\
D_{N} & {\left[\frac{\partial^{2} u_{r}}{\partial r^{2}}+\frac{\partial w}{\partial r} \frac{\partial^{2} w}{\partial r^{2}}+\frac{1}{r} \frac{\partial u_{r}}{\partial r}-\frac{1}{r^{2}} u_{r}+\frac{1+\mu}{2 r^{2}} \frac{\partial w}{\partial \theta} \frac{\partial^{2} w}{\partial r \partial \theta}-\frac{1+\mu}{2 r^{3}}\left(\frac{\partial w}{\partial \theta}\right)^{2}+\frac{1-\mu}{2 r}\left(\frac{\partial w}{\partial r}\right)^{2}\right] } \\
& +\sigma_{0} h B_{0 z}\left[\left(\frac{\partial w}{\partial t}+\Omega \frac{\partial w}{\partial \theta}\right) B_{0 r}-\frac{d u_{r}}{d t} B_{0 z}\right]+F_{0 r}=\rho h\left[\frac{d^{2} u_{r}}{d t^{2}}-\Omega^{2}\left(r+u_{r}\right)\right]
\end{aligned}
$$

in which $\rho$ denotes material density, $w$ - transverse displacement, $u_{r}$ - longitudinal displacement, $\sigma_{0}$ - electric conductance, the flexural rigidity is $D_{M}=E h^{3} /\left[12\left(1-\mu^{2}\right)\right]$, tensile rigidity $D_{N}=E h /\left(1-\mu^{2}\right), E$ denotes Young's modulus and $\mu$ denotes Poisson's ratio, 
It can be concluded that there are static load terms in the longitudinal vibration equation when the rotating annular plate is subjected to the radial load. It is assumed that the circular plate has the initial deflection $u_{0}$, and its deflection is $u_{1}$ when it vibrates. So, the total deflection $u_{r}$ can be written as

$$
u_{r}=u_{0}+u_{1}
$$

Then Eq. (2.2) can be rewritten as

$$
\begin{aligned}
D_{N} & {\left[\left(\frac{\partial^{2} u_{0}}{\partial r^{2}}+\frac{\partial^{2} u_{1}}{\partial r^{2}}\right)+\frac{\partial w}{\partial r} \frac{\partial^{2} w}{\partial r^{2}}+\frac{1}{r}\left(\frac{\partial u_{0}}{\partial r}+\frac{\partial u_{1}}{\partial r}\right)-\frac{1}{r^{2}}\left(u_{0}+u_{1}\right)\right.} \\
& \left.+\frac{1+\mu}{2 r^{2}} \frac{\partial w}{\partial \theta} \frac{\partial^{2} w}{\partial r \partial \theta}-\frac{1+\mu}{2 r^{3}}\left(\frac{\partial w}{\partial \theta}\right)^{2}+\frac{1-\mu}{2 r^{2}} \frac{\partial w}{\partial r} \frac{\partial^{2} w}{\partial \theta^{2}}+\frac{1-\mu}{2 r}\left(\frac{\partial w}{\partial r}\right)^{2}\right] \\
& +\sigma_{0} h B_{0 z}\left[\left(\frac{\partial w}{\partial t}+\Omega \frac{\partial w}{\partial \theta}\right) B_{0 r}-\frac{d u_{1}}{d t} B_{0 z}\right]+F_{0 r}=\rho h\left[\frac{d^{2} u_{1}}{d t^{2}}-\Omega^{2}\left(r+u_{0}+u_{1}\right)\right]
\end{aligned}
$$

The initial deflection $u_{0}$ satisfies the equation

$$
D_{N}\left(\frac{\partial^{2} u_{0}}{\partial r^{2}}+\frac{1}{r} \frac{\partial u_{0}}{\partial r}-\frac{1}{r^{2}} u_{0}\right)+F_{0}=-\rho h \Omega^{2}\left(r+u_{0}\right)
$$

The annular thin plate with inner-clamped and outer-free boundary conditions is analysed. Equation (2.4) can be solved by using the Galekin method in which we assume (Chonan et al., 1986)

$$
u_{0}=f_{0} \sum_{m=0}^{M}\left(\frac{r}{R}-\frac{r_{0}}{R}\right)^{m+1}\left(\frac{r}{R}-1\right)=f_{0} U_{m}
$$

Substituting Eq. (2.5) into Eq. (2.4), one can obtain

$$
f_{0}=-\frac{a_{2}}{a_{1}}
$$

We assumed that $w$ and additional deflection $u_{1}$ can be written as follows (Chonan et al., 1986)

$$
w=\sum_{n=0}^{N} W(r) T_{w n}(t) \cos (n \theta) \quad u_{1}=U_{m}(r) T_{u}(t)
$$

Let $M=0$ and $N=1$, then Eq. (2.7) is

$$
w=W\left(T_{w 0}+T_{w 1} \cos \theta\right) \quad u_{1}=U_{0} T_{u}
$$

Substituting Eq. (2.8) into Eqs. (2.1) and using the Galerkin method, resonance differential equations are obtained

$$
\begin{aligned}
& A_{11} \ddot{T}_{w 0}+A_{12} \dot{T}_{w 0}+\left(A_{13}+A_{14} F_{0 r}\right) T_{w 0}+A_{15} T_{w 0}^{3}+B_{16} T_{w 0} T_{w 1}^{2}+C_{11} \dot{T}_{u} \\
& \quad+C_{12} T_{w 0}\left(T_{u}+f_{0}\right)+A_{P} P_{z}=0 \\
& B_{21} \ddot{T}_{w 1}+B_{22} \dot{T}_{w 1}+\left(B_{23}+B_{24} F_{0 r}\right) T_{w 1}+B_{25} T_{w 1}^{3}+A_{26} T_{w 0}^{2} T_{w 1}+C_{23} T_{w 1}\left(T_{u}+f_{0}\right)=0 \\
& D_{11} \ddot{T}_{u}+D_{12} \dot{T}_{u}+D_{13} T_{u}+D_{14} T_{w 0}^{2}+D_{16} T_{w 1}^{2}+D_{18} \dot{T}_{w 0}-D_{F} F_{1} \cos \omega_{1} t=0
\end{aligned}
$$




\section{The theoretical solution of resonance by the combined parametric and forced excitation}

Let $P_{z}=P_{1} \cos \omega_{2} t$ and introduce nondimensional variables $\omega_{1} t=2 \tau, \omega_{2} t=\tau$ and a small parameter $\varepsilon$ to study weakly nonlinear oscillations. Equations (2.9) are rewritten as

$$
\begin{aligned}
& \ddot{q}_{0}+2 \varepsilon \zeta_{1} \dot{q}_{0}+\omega_{01}^{2} q_{0}+\varepsilon \alpha_{11} \cos (2 \tau) q_{0}+\varepsilon \alpha_{12} q_{0}^{3}+\varepsilon \alpha_{13} q_{0} q_{1}^{2}+\varepsilon \alpha_{14} \dot{q}_{u} \\
& \quad+\varepsilon \alpha_{15} q_{0}\left(q_{u}+f\right)+\varepsilon \alpha_{16} \cos \tau=0 \\
& \ddot{q}_{1}+2 \varepsilon \zeta_{2} \dot{q}_{1}+\omega_{02}^{2} q_{1}+\varepsilon \alpha_{21} \cos (2 \tau) q_{1}+\varepsilon \alpha_{22} q_{1}^{3}+\varepsilon \alpha_{23} q_{0}^{2} q_{1}+\varepsilon \alpha_{24} q_{1}\left(q_{u}+f\right)=0 \\
& \ddot{q}_{u}+2 \varepsilon \zeta_{3} \dot{q}_{u}+\omega_{03}^{2} q_{u}+\varepsilon \alpha_{31} q_{0}^{2}+\varepsilon \alpha_{32} q_{1}^{2}+\varepsilon \alpha_{33} \dot{q}_{0}=\varepsilon \alpha_{34} \cos (2 \tau)
\end{aligned}
$$

It can be noted from Eqs. (3.1) that the system possesses different resonance conditions when $\omega_{01}, \omega_{02}, \omega_{03}$ are respectively close to 1 . In the following Subsections, these resonance cases are discussed.

Case I: $\omega_{01} \approx 1$

We assume

$$
\omega_{01}=1+\varepsilon \sigma
$$

where $\sigma$ denotes the nondimensional detuning parameter. $\omega_{02}$ and $\omega_{03}$ can be written in the form

$$
\omega_{02}=\omega_{01} \sqrt{\frac{A_{11}\left(B_{23}+B_{24} F_{0}\right)}{B_{21}\left(A_{13}+A_{14} F_{0}\right)}}=g_{1} \omega_{01} \quad \omega_{03}=\omega_{01} \sqrt{\frac{A_{11} D_{13}}{D_{11}\left(A_{13}+A_{14} F_{0}\right)}}=g_{2} \omega_{01}
$$

By the use of the method of multiple scales (Chonan et al., 1986), the solutions to Eqs. (3.1) in terms of different time scales can be expressed as

$$
\begin{aligned}
& q_{0}(\tau, \varepsilon)=q_{01}\left(T_{0}, T_{1}\right)+\varepsilon q_{02}\left(T_{0}, T_{1}\right) \\
& q_{u}(\tau, \varepsilon)=q_{u 1}\left(T_{0}, T_{1}\right)+\varepsilon q_{u 2}\left(T_{0}, T_{1}\right)
\end{aligned} \quad q_{1}(\tau, \varepsilon)=q_{11}\left(T_{0}, T_{1}\right)+\varepsilon q_{12}\left(T_{0}, T_{1}\right)
$$

Substituting Eqs. (3.2) and (3.3) into Eqs. (3.1), and equating the coefficients of the same order of $\varepsilon$ in both sides, the following equations are obtained

$$
\begin{aligned}
& D_{0}^{2} q_{01}+q_{01}=0 \\
& D_{0}^{2} q_{02}+q_{02}=-2 D_{0} D_{1} q_{01}-2 \zeta_{1} D_{0} q_{01}-2 \sigma q_{01}-\alpha_{12} q_{01}^{3}-\alpha_{13} q_{01} q_{11}^{2}-\alpha_{14} D_{0} q_{u 1} \\
& \quad-\alpha_{15} q_{01}\left(q_{u 1}+f\right)-\alpha_{11} \cos (2 \tau) q_{01}-\alpha_{16} \cos \tau \\
& D_{0}^{2} q_{11}+g_{1}^{2} q_{11}=0 \\
& D_{0}^{2} q_{12}+g_{1}^{2} q_{12}=-2 D_{0} D_{1} q_{11}-2 \zeta_{2} D_{0} q_{11}-2 \sigma g_{1}^{2} q_{11}-\alpha_{22} q_{11}^{3}-\alpha_{23} q_{01}^{2} q_{11} \\
& \quad-\alpha_{24} q_{11}\left(q_{u 1}+f\right)-\alpha_{21} \cos (2 \tau) q_{11} \\
& D_{0}^{2} q_{u 1}+g_{2}^{2} q_{u 1}=0 \\
& D_{0}^{2} q_{u 2}+g_{2}^{2} q_{u 2}=\alpha_{34} \cos (2 \tau)-2 D_{0} D_{1} q_{u 1}-2 \zeta_{3} D_{0} q_{u 1}-2 \sigma g_{2}^{2} q_{u 1} \\
& \quad-\alpha_{13} q_{01}^{2}-\alpha_{32} q_{11}^{2}-\alpha_{33} D_{0} q_{01}
\end{aligned}
$$

where $D_{0}=\partial / \partial T_{0}, D_{1}=\partial / \partial T_{1}$. The solutions to Eq. (3.4) $)_{1}$, Eq. (3.4) $)_{3}$ and Eq. $(3.4)_{5}$ are

$$
\begin{aligned}
& q_{01}=S_{1}\left(T_{1}\right) \exp \left(\mathrm{i} T_{0}\right)+\bar{S}_{1}\left(T_{1}\right) \exp \left(-\mathrm{i} T_{0}\right) \\
& q_{11}=S_{2}\left(T_{1}\right) \exp \left(\mathrm{i} g_{1} T_{0}\right)+\bar{S}_{2}\left(T_{1}\right) \exp \left(-\mathrm{i} g_{1} T_{0}\right) \\
& q_{u 1}=S_{3}\left(T_{1}\right) \exp \left(\mathrm{i} g_{2} T_{0}\right)+\bar{S}_{3}\left(T_{1}\right) \exp \left(-\mathrm{i} g_{2} T_{0}\right)
\end{aligned}
$$


Substituting Eqs. (3.5) into Eq. (3.4) $)_{2}$, Eq. $(3.4)_{4}$ and Eq. $(3.4)_{6}$, because of $g_{1} \neq g_{2} \neq 1$, we let the coefficients of $\exp \left(\mathrm{i} T_{0}\right)$ vanish. This gives

$$
\begin{aligned}
& -2 \mathrm{i} S_{1}^{\prime} \exp \left(\mathrm{i} T_{0}\right)-2 \mathrm{i} \zeta_{1} S_{1} \exp \left(\mathrm{i} T_{0}\right)-2 \sigma S_{1} \exp \left(\mathrm{i} T_{0}\right)-3 \alpha_{12} S_{1}^{2} \bar{S}_{1} \exp \left(\mathrm{i} T_{0}\right) \\
& \quad-2 \alpha_{13} S_{1} S_{2} \bar{S}_{2} \exp \left(\mathrm{i} T_{0}\right)+\alpha_{15} S_{1} f \exp \left(\mathrm{i} T_{0}\right)-\frac{1}{2} \alpha_{11} \bar{S}_{1} \exp \left(\mathrm{i} T_{0}\right)-\frac{1}{2} \alpha_{16} \exp \left(\mathrm{i} T_{0}\right)=0 \\
& -2 \mathrm{i} g_{1} S_{2}^{\prime} \exp \left(\mathrm{i} g_{1} T_{0}\right)-2 \mathrm{i} g_{1} \zeta_{2} S_{2} \exp \left(\mathrm{i} g_{1} T_{0}\right)-2 \sigma g_{1}^{2} S_{2} \exp \left(\mathrm{i} g_{1} T_{0}\right) \\
& \quad-3 \alpha_{22} S_{2}^{2} \bar{S}_{2} \exp \left(\mathrm{i} g_{1} T_{0}\right)-2 \alpha_{23} S_{1} \bar{S}_{1} S_{2} \exp \left(\mathrm{i} g_{1} T_{0}\right)=0 \\
& -2 \mathrm{i} g_{2} S_{3}^{\prime} \exp \left(\mathrm{i} g_{2} T_{0}\right)-2 \mathrm{i} g_{2} \zeta_{3} S_{3} \exp \left(\mathrm{i} g_{2} T_{0}\right)-2 \sigma g_{2}^{2} S_{3} \exp \left(\mathrm{i} g_{2} T_{0}\right)=0
\end{aligned}
$$

Putting $S_{n}=0.5 a_{2}\left(T_{1}\right) \exp \left[\mathrm{i} \beta_{2}\left(T_{1}\right)\right], n=1,2,3$ in Eqs. (3.6) and separating the real and imaginary terms, one can see that only Eq. $(3.6)_{1}$ has non-zero amplitude solutions. We have $a_{1}^{\prime}=0, \beta_{1}^{\prime}=0$ due to steady-state motion of the system. Hence, we conclude that

$$
\begin{aligned}
& \zeta_{1} a_{1}=\frac{1}{4} \alpha_{11} a_{1} \sin \left(2 \beta_{1}\right)+\frac{1}{2} \alpha_{16} \sin \beta_{1} \\
& -\left(a_{1} \sigma+\frac{3}{8} \alpha_{12} a_{1}^{3}\right)=\frac{1}{2} \alpha_{15} f a_{1}+\frac{1}{4} \alpha_{11} a_{1} \cos \left(2 \beta_{1}\right)+\frac{1}{2} \alpha_{16} \cos \beta_{1}
\end{aligned}
$$

In order to solve Eq. (3.7), we assume

$$
M=a_{1} \cos \beta_{1} \quad N=a_{1} \sin \beta_{1}
$$

We have $M^{\prime}=0$ and $N^{\prime}=0$ due to steady-state motion of the system. $M$ and $N$ can be given by

$$
\begin{aligned}
& M=\frac{\alpha_{16}\left(\sigma-\frac{1}{4} \alpha_{11}+\frac{3}{8} \alpha_{12} a_{1}^{2}-\frac{1}{2} \alpha_{15} f\right)}{2\left[\zeta_{1}^{2}+\left(\sigma+\frac{3}{8} \alpha_{12} a_{1}^{2}-\frac{1}{2} \alpha_{15} f-\frac{1}{4} \alpha_{11}\right)\left(\sigma+\frac{3}{8} \alpha_{12} a_{1}^{2}-\frac{1}{2} \alpha_{15} f+\frac{1}{4} \alpha_{11}\right)\right]} \\
& N=\frac{-\alpha_{16} \zeta_{1}}{2\left[\zeta_{1}^{2}+\left(\sigma+\frac{3}{8} \alpha_{12} a_{1}^{2}-\frac{1}{2} \alpha_{15} f-\frac{1}{4} \alpha_{11}\right)\left(\sigma+\frac{3}{8} \alpha_{12} a_{1}^{2}-\frac{1}{2} \alpha_{15} f+\frac{1}{4} \alpha_{11}\right)\right]}
\end{aligned}
$$

According to $M^{2}+N^{2}=a_{1}^{2}$, the non-axisymmetric vibration differential equation by the combined parametric and forced excitations of the rotating annular plate is given by

$$
\begin{aligned}
& \alpha_{16}^{2}\left(\sigma-\frac{1}{4} \alpha_{11}+\frac{3}{8} \alpha_{12} a_{1}^{2}-\frac{1}{2} \alpha_{15} f\right)^{2}+\left(\alpha_{16} \zeta_{1}\right)^{2} \\
& \quad=4 a_{1}^{2}\left[\zeta_{1}^{2}+\left(\sigma+\frac{3}{8} \alpha_{12} a_{1}^{2}-\frac{1}{2} \alpha_{15} f-\frac{1}{4} \alpha_{11}\right)\left(\sigma+\frac{3}{8} \alpha_{12} a_{1}^{2}-\frac{1}{2} \alpha_{15} f+\frac{1}{4} \alpha_{11}\right)\right]^{2}
\end{aligned}
$$

Case II: $\omega_{02} \approx 1$

We assume

$$
\omega_{02}=1+\varepsilon \sigma
$$

where $\omega_{02}$ and $\omega_{03}$ can be written in the form

$$
\omega_{01}=\omega_{02} \sqrt{\frac{B_{21}\left(A_{13}+A_{14} F_{0}\right)}{A_{11}\left(B_{23}+B_{24} F_{0}\right)}}=\omega_{02} h_{1} \quad \omega_{03}=\omega_{02} \sqrt{\frac{B_{21} D_{13}}{D_{11}\left(B_{23}+B_{24} F_{0}\right)}}=\omega_{02} h_{2}
$$


By the use of the method of multiple scales, substituting Eqs. (3.11) and Eqs. (3.2) and $(3.3)_{1,2}$ into Eqs. (3.1), we equate the coefficients of the same order of $\varepsilon$ in both sides. According to case I, we get the following conditions to avoid the occurrence of duration items

$$
\begin{gathered}
-2 \mathrm{i} S_{2}^{\prime} \exp \left(\mathrm{i} T_{0}\right)-2 \mathrm{i} \zeta_{2} S_{2} \exp \left(\mathrm{i} T_{0}\right)-2 \sigma g_{1}^{2} S_{2} \exp \left(\mathrm{i} T_{0}\right)-3 \alpha_{22} S_{2}^{2} \bar{S}_{2} \exp \left(\mathrm{i} T_{0}\right) \\
-2 \alpha_{23} S_{1} \bar{S}_{1} S_{2} \exp \left(\mathrm{i} T_{0}\right)+\alpha_{24} S_{2} f \exp \left(\mathrm{i} T_{0}\right)-\frac{1}{2} \alpha_{21} \bar{S}_{2} \exp \left(\mathrm{i} T_{0}\right)=0
\end{gathered}
$$

Putting $S_{2}=0.5 a_{2}\left(T_{1}\right) \exp \left[\mathrm{i} \beta_{2}\left(T_{1}\right)\right]$ in Eq. (3.12) and eparating the real and imaginary terms, we have $a_{2}^{\prime}=0, \beta_{2}^{\prime}=0$ due to steady-state motion of the system. The frequency-response equation can be obtained

$$
\left(a_{2} \sigma+\frac{3}{8} \alpha_{22} a_{2}^{3}-\frac{1}{2} \alpha_{24} f a_{2}\right)^{2}+\left(\zeta_{2} a_{2}\right)^{2}=\left(\frac{1}{4} \alpha_{21} a_{2}\right)^{2}
$$

Case III: $\omega_{03} \approx 1$

We assume

$$
\omega_{03}=1+\varepsilon \sigma
$$

From Eq. (2.23), we can see that in this case the system has the main resonance. We put $\omega_{1} t=\tau$ and rewrite nondimensional Eqs. (2.9), which gives

$$
\begin{aligned}
& \ddot{q}_{0}+2 \widetilde{\zeta}_{1} \dot{q}_{0}+\omega_{01}^{2} q_{0}+\widetilde{\alpha}_{11} \cos (\tau) q_{0}+\widetilde{\alpha}_{12} q_{0}^{3}+\widetilde{\alpha}_{13} q_{0} q_{1}^{2}+\widetilde{\alpha}_{14} \dot{q}_{u}+\widetilde{\alpha}_{15} q_{u} q_{0}+\widetilde{\alpha}_{16} \cos \tau=0 \\
& \ddot{q}_{1}+2 \widetilde{\zeta}_{2} \dot{q}_{1}+\omega_{02}^{2} q_{1}+\widetilde{\alpha}_{21} \cos (\tau) q_{1}+\widetilde{\alpha}_{22} q_{1}^{3}+\widetilde{\alpha}_{23} q_{0}^{2} q_{1}+\widetilde{\alpha}_{24} q_{u} q_{1}=0 \\
& \ddot{q}_{u}+2 \widetilde{\zeta}_{3} \dot{q}_{u}+\omega_{03}^{2} q_{u}+\widetilde{\alpha}_{31} q_{0}^{2}+\widetilde{\alpha}_{32} q_{1}^{2}+\widetilde{\alpha}_{33} \dot{q}_{0}=\widetilde{\alpha}_{34} \cos \tau
\end{aligned}
$$

where $\omega_{02}$ and $\omega_{03}$ can be written in the form

$$
\omega_{01}=\omega_{03} \sqrt{\frac{D_{11}\left(A_{13}+A_{14} F_{0}\right)}{D_{13} A_{11}}}=j_{1} \omega_{03} \quad \omega_{02}=\omega_{03} \sqrt{\frac{\left(B_{23}+B_{24} F_{0}\right) D_{11}}{D_{13} B_{21}}}=j_{2} \omega_{03}
$$

By the use of the method of multiple scales, substituting Eqs. (3.14) and Eqs. (3.3) into Eqs. (3.15), we equate the coefficients of the same order of $\varepsilon$ in both sides. According to case I, we get the following conditions to avoid the occurrence of duration terms

$$
\frac{1}{2} \alpha_{34} \exp \left(\mathrm{i} T_{0}\right)-2 \mathrm{i} S_{3}^{\prime} \exp \left(\mathrm{i} T_{0}\right)-2 \mathrm{i} \zeta_{3} S_{3} \exp \left(\mathrm{i} T_{0}\right)-2 \sigma S_{3} \exp \left(\mathrm{i} T_{0}\right)-\mathrm{i} \alpha_{33} S_{1} \exp \left(\mathrm{i} T_{0}\right)=0
$$

Substituting $S_{3}=0.5 a_{3}\left(T_{1}\right) \exp \left[\mathrm{i} \beta_{3}\left(T_{1}\right)\right]$ into Eq. (3.16) and separating the real and imaginary terms, we have $a_{3}^{\prime}=0, \beta_{3}^{\prime}=0$ due to steady-state motion of the system. Hence, we get

$$
a_{3}=\frac{\alpha_{34}}{2 \sqrt{\sigma^{2}+\xi_{3}^{2}}}
$$

\section{Numerical results and discussions}

In the numerical study of the magneto-elastic combination resonance of a copper annular plate with internal-clamped and external-free boundary conditions, the circular plate with the following parameters is used: electric conductivity $\sigma_{0}=5.7143 \cdot 10^{7}(\Omega \mathrm{m})^{-1}$, Young's modulus $E=108 \mathrm{GPa}$, Poisson's ratio $\mu=0.33$, density $\rho=8920 \mathrm{~kg} / \mathrm{m}^{3}$ and $\Omega=5000 \mathrm{r} / \mathrm{min}$. 
Case I: $\omega_{01} \approx 1$

Figures 2-5 show the response curves of amplitude $a_{1}$ in relation to the frequency detuning parameter $\varepsilon \sigma$ when $\omega_{01} \approx 1$. We note that there are two peaks of the resonance in the figures, and complex multivalued variations occurred.

Figure $2\left(r_{0}=0.3 \mathrm{~m}, R=0.8 \mathrm{~m}, h=4 \mathrm{~mm}, B_{0 r}=0.06 \mathrm{~T}, B_{0 z}=1 \mathrm{~T}, F_{1}=1 \mathrm{kN} / \mathrm{m}\right.$, $P_{1}=130 \mathrm{~N} / \mathrm{m}^{2}$ ) indicates that the resonance amplitude of the system decreases gradually, the distance between the two resonance regions increases slightly and the peak value of the left resonance region extends to the right with an increase in the radial load $F_{0}$. As the radial load $F_{0}$ continues to increase, the number of solutions of the system will be reduced from five to three, and the resonance amplitude of the system increases with an increase in $F_{0}$.

(a)

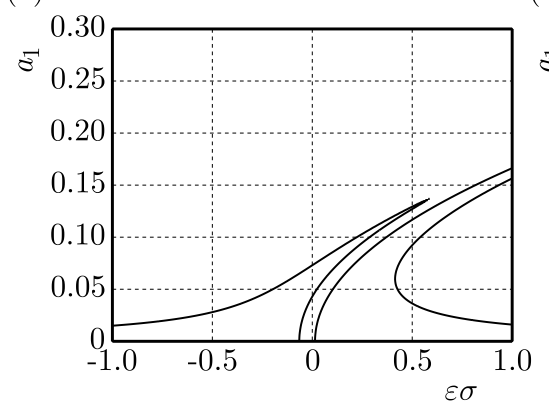

(b)

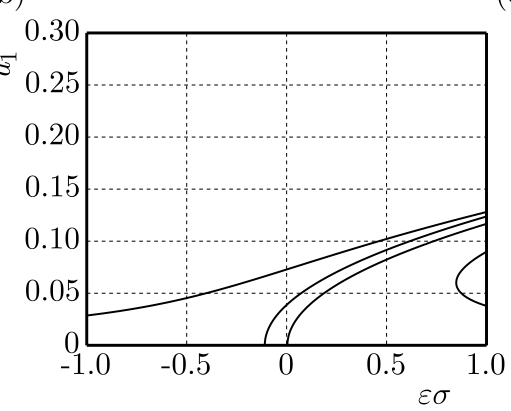

(c)

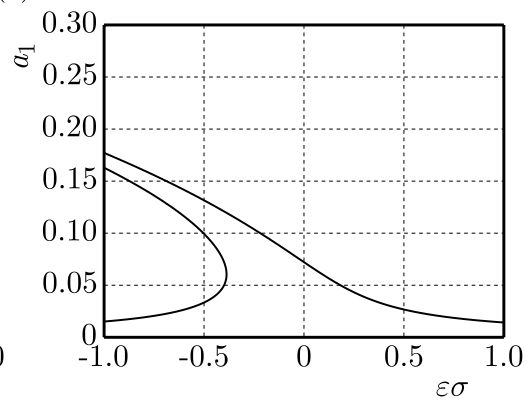

Fig. 2. Amplitude-frequency reponse curve for different $F_{0}$ : (a) $F_{0}=10 \mathrm{kN} / \mathrm{m}$, (b) $F_{0}=15 \mathrm{kN} / \mathrm{m}$, (c) $F_{0}=30 \mathrm{kN} / \mathrm{m}$

Figure $3\left(r_{0}=0.3 \mathrm{~m}, R=0.8 \mathrm{~m}, h=4 \mathrm{~mm}, B_{0 r}=0.06 \mathrm{~T}, B_{0 z}=1 \mathrm{~T}, F_{0}=10 \mathrm{kN} / \mathrm{m}\right.$, $P_{1}=130 \mathrm{~N} / \mathrm{m}^{2}$ ) shows that the multivalue property of the system has changed greatly with the increase of the radial load $F_{1}$. The peak value of the left resonance region is obviously reduced. The right resonance curve has an intersection point: the resonance amplitude on the left side of the intersection point gradually increases, and the resonance amplitude on the right side of the intersection point gradually decreases. The width of the right resonance region increases gradually. The size of the zero solution region between the two resonance peaks does not change and the position moves to the left.

(a)

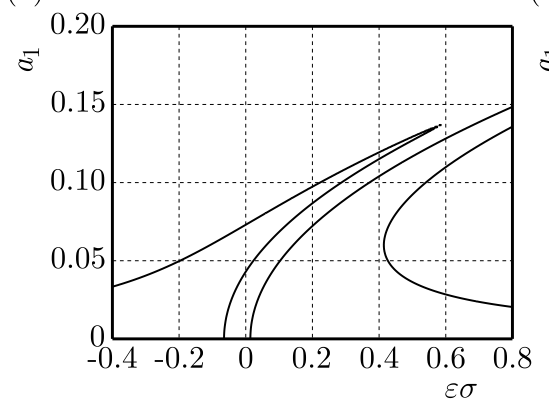

(b)

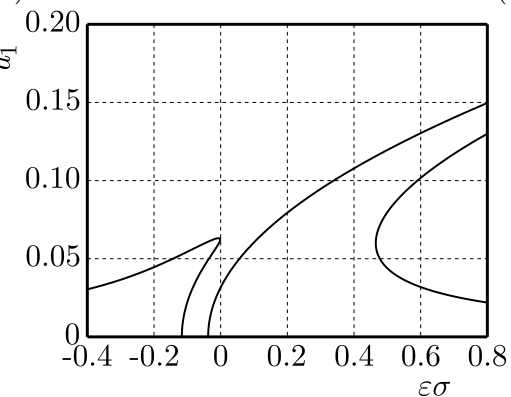

(c)

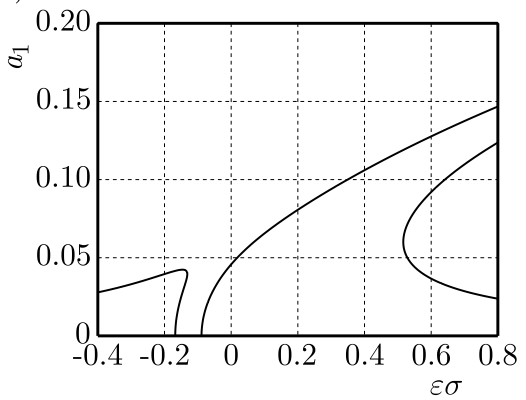

Fig. 3. Amplitude-frequency reponse curve for different $F_{1}$ : (a) $F_{1}=1 \mathrm{kN} / \mathrm{m}$, (b) $F_{0}=3 \mathrm{kN} / \mathrm{m}$, (c) $F_{0}=5 \mathrm{kN} / \mathrm{m}$

Figure $4\left(r_{0}=0.3 \mathrm{~m}, R=0.8 \mathrm{~m}, h=4 \mathrm{~mm}, B_{0 r}=0.06 \mathrm{~T}, B_{0 z}=1 \mathrm{~T}, F_{0}=10 \mathrm{kN} / \mathrm{m}\right.$, $\left.F_{1}=1 \mathrm{kN} / \mathrm{m}\right)$ shows that with an increase in the excitation force $P_{1}$, the amplitude of the resonance region on the left side of the system increases gradually, the amplitude of the resonance region on the right side decreases gradually, and the two resonance regions gradually expand. 
(a)

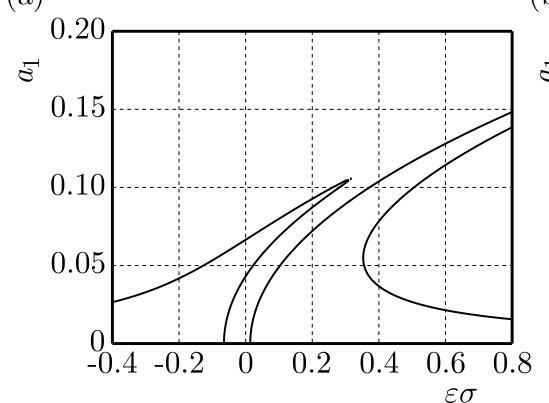

(b)

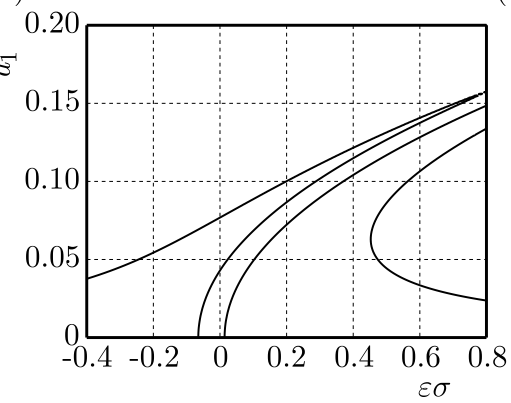

(c)

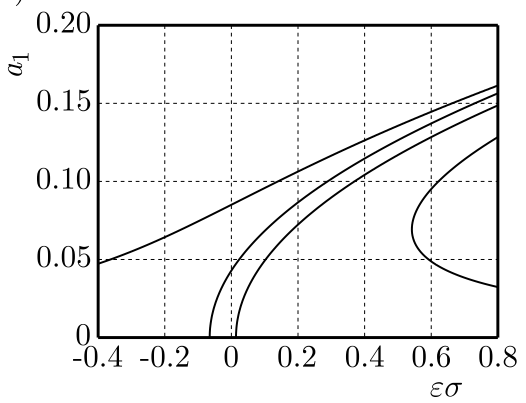

Fig. 4. Amplitude-frequency reponse curve for different $P_{1}$ : (a) $P_{1}=100 \mathrm{~N} / \mathrm{m}^{2}$, (b) $P_{1}=150 \mathrm{~N} / \mathrm{m}^{2}$, (c) $P_{1}=200 \mathrm{~N} / \mathrm{m}^{2}$

Figure $5 \mathrm{a}\left(r_{0}=0.3 \mathrm{~m}, R=0.8 \mathrm{~m}, h=4 \mathrm{~mm}, B_{0 z}=1 \mathrm{~T}, F_{0}=10 \mathrm{kN} / \mathrm{m}, F_{1}=1 \mathrm{kN} / \mathrm{m}\right.$, $P_{1}=130 \mathrm{~N} / \mathrm{m}^{2}$ ) shows the response curves of the amplitude versus magnetic induction intensity $B_{0 r}$. It can be seen that there are three steady-state solutions when $B_{0 r}$ equals zero, the number of steady-state solution increases to five when $B_{0 r}$ increases to $0.01 \mathrm{~T}$. As the magnetic induction intensity $B_{0 r}$ increases to $0.04 \mathrm{~T}$, the number of steady-state solutions is reduced to three, and the resonance amplitude decreases slightly. Figure $5 \mathrm{~b}\left(r_{0}=0.3 \mathrm{~m}, R=0.8 \mathrm{~m}\right.$, $h=4 \mathrm{~mm}, B_{0 r}=0.02 \mathrm{~T}, F_{0}=10 \mathrm{kN} / \mathrm{m}, F_{1}=1 \mathrm{kN} / \mathrm{m}, P_{1}=130 \mathrm{~N} / \mathrm{m}^{2}$ ) shows the response curves of the amplitude versus magnetic induction intensity $B_{0 z}$. It can be seen that there are five steady-state solutions when $B_{0 z}$ equals zero, which is due to the result of $B_{0 r}=0.02 \mathrm{~T}$. With the increase of $B_{0 z}$, the number of steady-state solutions decreases to three, and the amplitude decreases slightly. Figure $5 \mathrm{c}\left(F_{1}=1 \mathrm{kN} / \mathrm{m}, P_{1}=130 \mathrm{~N} / \mathrm{m}^{2}, B_{0 r}=0.02 \mathrm{~T}, B_{0 r}=0.02 \mathrm{~T}\right.$, $\left.F_{0}=10 \mathrm{kN} / \mathrm{m}, r_{0}=0.3 \mathrm{~m}, h=4 \mathrm{~mm}\right)$ shows the response curves of the amplitude versus the ratio $\psi$ of the outer to inner diameter when $R=\psi r_{0}$ is assumed. It can be seen from the diagram that with the increase of the ratio $\psi$ from 2 , there are three steady-state solutions in the system, and the amplitude firstly decreases and then increases. The resonance occurs near $\psi=2.4$. As $\psi$ continues to increase, the amplitude of the system gradually decreases and tends to stabilize. The number of steady-state solutions increases from three to five. When $\psi$ is greater than 3.3, the number of amplitudes is reduced to three.

(a)

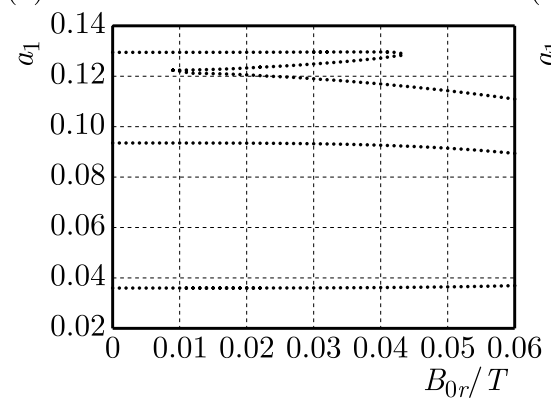

(b)

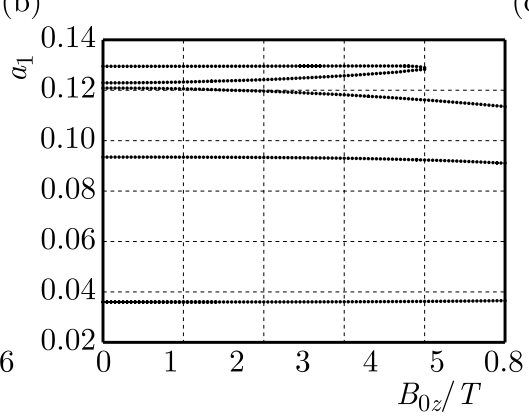

(c)

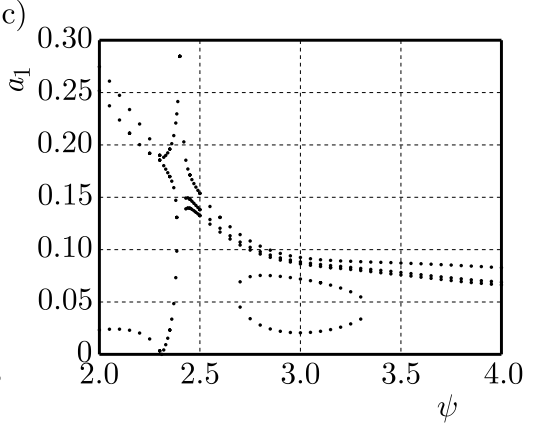

Fig. 5. Amplitude-magnetic induction intensity: (a) $B_{0 r}$ curve, (b) $B_{0 z}$ curve. (c) Amplitude-ratio of the external and internal diameters curve

Case II: $\omega_{02} \approx 1$

Figures 6 and 7 show the response curves of amplitude $a_{2}$ in relation to the frequency detuning parameter $\varepsilon \sigma$ when $\omega_{02} \approx 1$. With an increase in the coordination parameter $\varepsilon \sigma$ from left to right, the amplitude gradually decreases to zero and the resonance disappears.

Figure 6 a shows that the amplitude gradually increases, and the vanishing point of amplitude gradually moves away from the zero point of the transverse axis with the increase of magnetic 
induction intensity $B_{0 r}$. Figure $6 \mathrm{~b}$ shows that there exists an intersection point for the stable solution of different radial loads $F_{0}$ in the graph. With the increase of the radial load $F_{0}$, the amplitude of the stable solution increases gradually on the left side of the intersection point, and the amplitude of the stable solution decreases gradually on the right side of the intersection point. The vanishing point of the amplitude gradually approaches the zero point of the horizontal axis with the increase of $F_{0}$, that is, the resonance region between the stable and unstable solutions decreases gradually. Figure $6 \mathrm{c}$ shows that with the increase of the radial load $F_{1}$, the amplitude of the system increases, and the vanishing point of the amplitude is far away from the zero point of the horizontal axis.

(a)

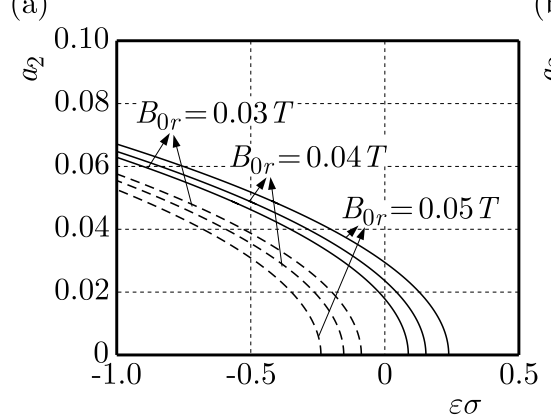

(b)

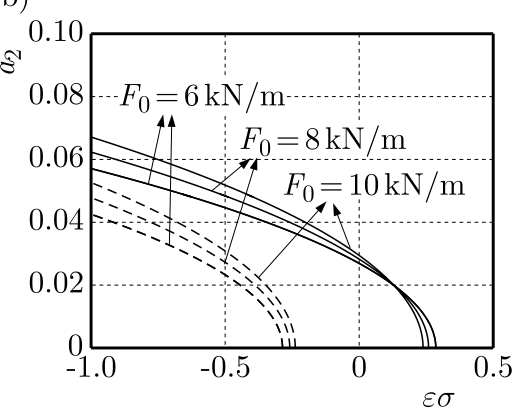

(c)

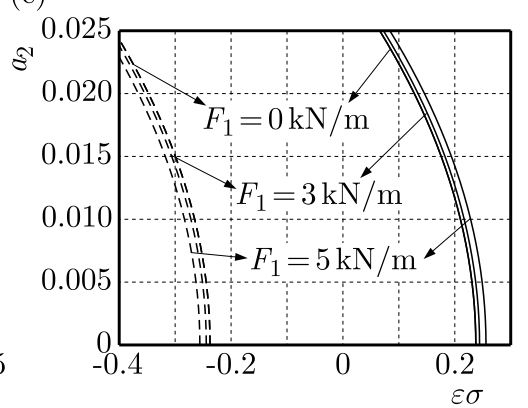

Fig. 6. Amplitude-frequency reponse curves for different: (a) $B_{0 r}$, (b) $F_{0}$, (c) $F_{1}$

Figure 7a shows that the unstable solutions of different plate thicknesses have an intersection point for each two branches. With the increase of plate thickness, the amplitude of the non-stable solution decreases gradually on the left side of the intersection point, the amplitude of the non-stable solutions increases gradually on the right side of the intersection point, and the amplitude of the stability solution decreases gradually. The vanishing point of the amplitude gradually approaches the zero point of the horizontal axis with the increase of $F_{0}$, that is, the resonance region between the stable and unstable solutions decreases gradually. Figute $7 \mathrm{~b}$ shows that the stable solutions with different inner diameters $r_{0}$ have an intersection in the graph. With the increase of the inner diameter, the amplitude of the stable solution on the left side of the intersection point increases gradually, the amplitude of the stable solution on the right side of the intersection point decreases gradually, and the amplitude of the unstable solution of the system increases gradually.

(a)

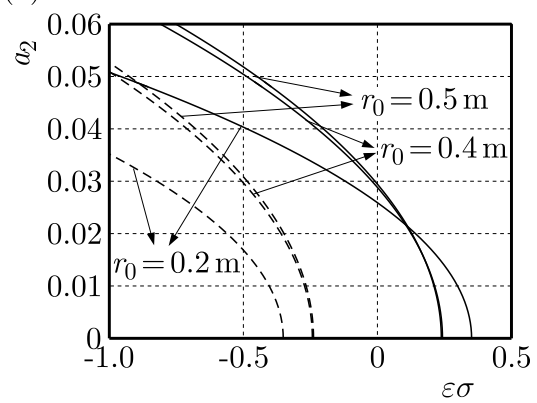

(b)

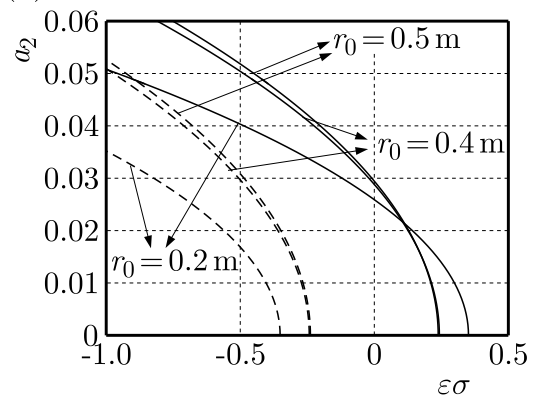

Fig. 7. Amplitude-frequency reponse curves for different: (a) $h$, (b) $r_{0}$

Case III: $\omega_{03} \approx 1$

Figures 8 and 9 show the response curves of amplitude $a_{3}$ varying with the frequency detuning parameter $\varepsilon \sigma$ when $\omega_{03} \approx 1$. It can be seen that with a change of the parameters, the variation of the amplitude of the system is small. Figures 8 and 9a show that with an increase in the 
magnetic induction intensity $B_{0 z}$, radial load $F_{0}$, outer diameter of the annular plate $R$ and a decrease in the radial load $F_{1}$, the radial amplitude of the annular plate increases gradually.

(a)

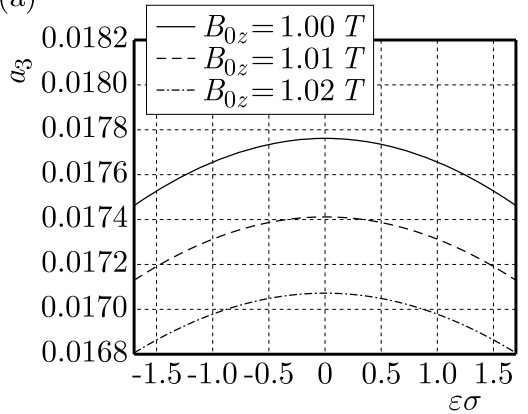

(b)

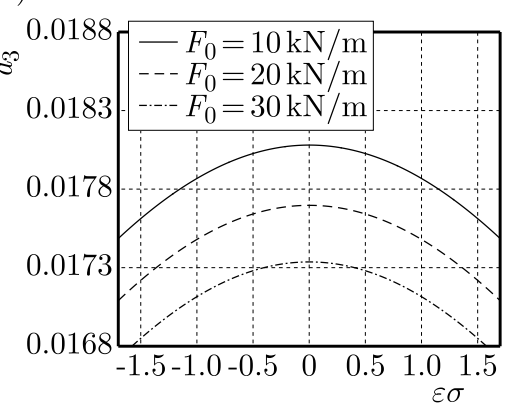

(c)

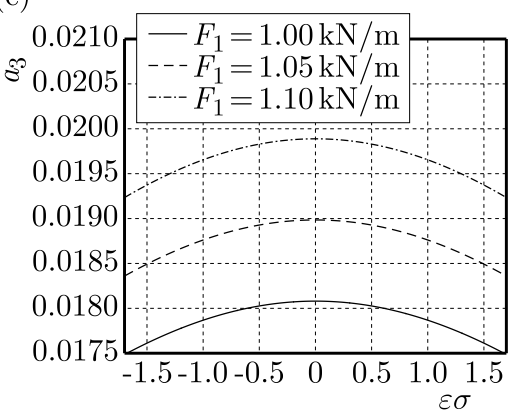

Fig. 8. Amplitude-frequency reponse curves for different: (a) $B_{0 z}$, (b) $F_{0}$, (c) $F_{1}$

(a)

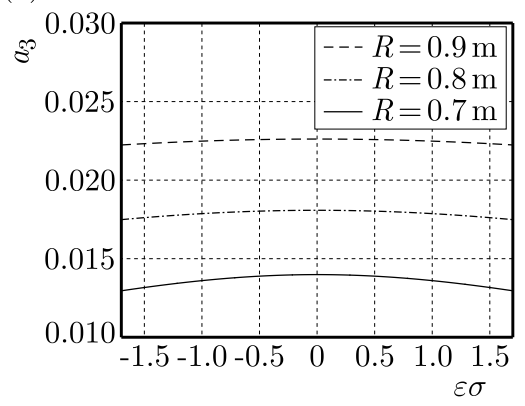

(b)

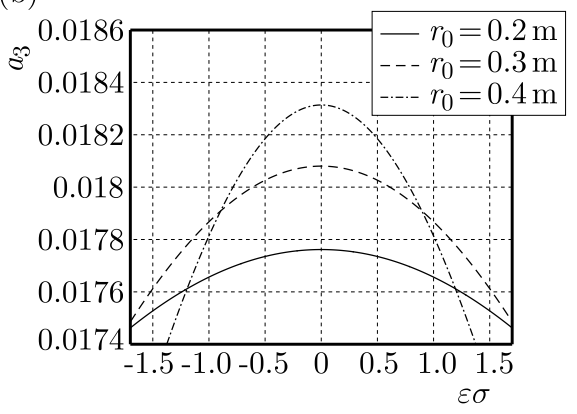

Fig. 9. Amplitude-frequency reponse curves for different: (a) $R$, (b) $r_{0}$

From Fig. 9b, we can find that with an increase in of the inner diameter $r_{0}$ of the annular plate, the resonance region of the system becomes narrower and any two amplitude frequency curves disclose two intersecting points: the amplitude of the region between two intersecting points increases with the increase of $r_{0}$, and the amplitude in other regions out of the region of the intersecting points decrease with the increase of $r_{0}$.

\section{Conclusions}

In this paper, the parameter principal resonance for multi-dimensional vibration of a rotating annular plate subjected to parametric excitation and forced excitation in a bidirectional magnetic field is studied, and vibration differential equations with a static load term are derived. The amplitude-frequency response equations of the system for different cases are obtained by using the multi-scale method, and the effects of the variable parameters on the resonance amplitude of the system in the corresponding cases are discussed. The results have shown that:

- When the resonance response occurs in the first order mode, the amplitude and its multi-variate properties of the system exhibit complicated changes with different parameters.

- When the resonance response occurs in the second order mode, the response is similar to the principal parameter resonance. The resonance amplitude of the system changes with different parameters.

- When the longitudinal response occurs, the response is similar to the primary resonance. With an increase in the magnetic induction intensity, radial load, external diameter of the annular plate and a decrease in the disturbance of the radial load, the amplitude of the system increases gradually. When thickness of the plate changes, the amplitude-frequency 
curve shows the intersection point, and the amplitude changes with different regularity on both sides of the intersection point.

\section{Acknowledgment}

This work was supported by the National Natural Science Foundation of China (No. 11472239), Natural Science Foundation of Hebei Province (No. A2015203023) and Doctoral Foundation of Yanshan University (No. BL19001).

\section{References}

1. Awrejcewicz J., Krysko V.A., Krysko A.V., 2004, Complex parametric vibrations of flexible rectangular plates, Meccanica, 39, 3, 221-244

2. Awrejcewicz J., Krysko V.A., Moldenkova T., 2006, Mathematical model of dissipative parametric vibrations of flexible plates with nonhomogeneous boundary conditions, Mathematical Problems in Engineering, DOI: 10.1155/MPE/2006/85623

3. Chen Y.R., Chen L.W., 2007, Vibration and stability of rotating polar orthotropic sandwich annular plates with a viscoelastic core layer, Composite Structures, 78, 1, 45-57

4. Chonan S., Mikami T., Ishikawa H., 1986, The vibrations and critical speeds of rotating sawblades, The Japan Society of Mechanical Engineers, 52, 478, 1805-1812

5. Hu Y.D., Li Z., 2017, Electromagnetic elastic coupling vibration equations of a conductive rotating circular plate, Chinese Journal of Applied Mechanics, 34, 1, 38-42

6. Hu Y.D., LI W.Q., 2018, Study on primary resonance and bifurcation of a conductive circular plate rotating in air-magnetic fields, Nonlinear Dynamic, 93, 2, 671-687

7. Hu Y.D., Li Z., Du G.J. Wang Y.N., 2018a, Magneto-elastic combination resonance of rotating circular plate with varying speed under alternating load, International Journal of Structural Stability and Dynamics, 18, 3, 1850032

8. Hu Y.D., Rong Y.T., Li J., 2018b, Primary parametric resonance of an axially accelerating beam subjected to static loads, Journal of Theoretical and Applied Mechanics, 56, 3, 815-828

9. Hu Y.D., WANG T., 2016, Nonlinear free vibration of a rotating circular plate under the static load in magnetic field, Nonlinear Dynamics, 85, 3, 1825-1835

10. Hu Y.D., Zhang J.Z., 2013, Principal parametric resonance of axially accelerating rectangular thin plate in magnetic field, Applied Mathematics and Mechanics, English Edition, 34, 11, 14051420

11. KANG J.H., 2017, Axisymmetric vibration of rotating annular plate with variable thickness subjected to tensile centrifugal body force, International Journal of Structural Stability and Dynamics, $\mathbf{1 7}, 9,1750101$

12. Li Z., HU Y.D., LI J., 2017, Magnetoelastic principal parametric resonance of a rotating electroconductive circular plate, Shock and Vibration, DOI: 10.1155/2017/5196847

13. Ma N.J., WANG R.H., HaN Q., 2014, Primary parametric resonance-primary resonance response of stiffened plates with moving boundary conditions, Nonlinear Dynamics, 79, 3, 2207-2223

14. Maretic R., Glavardanov V., Radomirovic D., 2007, Asymmetric vibrations and stability of a rotating annular plate loaded by a torque, Meccanica, 42, 6, 537-546

15. NARAin S., SRIVAStaVA H.K., 2004, Magnetoelastic torsional vibration of non-homogeneous aeolotropic cylindrical shell of viscoelastic solids, Defence Science Journal, 54, 4, 443-454

16. NAYfeh A.H., Mook D.T., 1979, Nonlinear Oscillations, John Wiley \& Sons, New York

17. Niu W.J., Zhang N.M., YAn X.P., YANG G.T., 2012, Nonlinear vibration of rectangular plate under the parametric excitation, Journal of Vibroengineering, 14, 3, 1076-1084 
18. Tylikowski A., Frischmuth K., 2003, Stability and stabilization of circular plate parametric vibrations, International Journal of Solids and Structures, 40, 19, 5187-5196

19. Wu Q., Takahashi K., Chen B., 2007, Influence of cable loosening on nonlinear parametric vibrations of inclined cables, Structural Engineering and Mechanics, 25, 2, 219-237

20. Xia Y., Wu Q., Xu Y., Fujino Y., Zhou X.Q., 2011, Verification of a cable element for cable parametric vibration of one-cable-beam system subject to harmonic excitation and random excitation, Advances in Structural Engineering, 14, 3, 589-595

21. YEH J.Y., 2011, Free vibration analysis of rotating polar orthotropic annular plate with ER damping treatment, Composites, Part B - Engineering, 42, 4, 781-788

22. Younesian D., Aleghafourian M.H., Esmailzadeh E., 2015, Vibration analysis of circular annular plates subjected to peripheral rotating transverse loads, Journal of Vibration and Control, 21, 7, 1443-1455

23. Zhang W., Huo Q.Z., 1991, Bifurcations of nonlinear oscillation system under combined parametric and forcing excitation, Acta Mechanica Sinica, 23, 4, 464-474

Manuscript received January 16, 2019; accepted for print May 6, 2019 